\title{
Visualization of Charge Carrier Trapping in Silicon at the Atomic Surface Level Using 4D Electron Imaging
}

Basamat S. Shaheen, Ahmed M. El-Zohry, Jun Yin, Michele De Bastiani, Stefaan De Wolf, Osman M. Bakr, Omar F. Mohammed*

King Abdullah University of Science and Technology (KAUST), Division of Physical Sciences and Engineering, Thuwal 23955-6900, KSA.

\section{Corresponding Author}

*omar.abdelsaboor@kaust.edu.sa

\section{ABSTRACT}

The ultrathin thickness $(\sim 1-2 \mathrm{~nm})$ of native oxide layer on silicon surfaces which acts as efficient trapping centers precludes the possibility of studying its impact on the surface-charge carrier dynamics by conventional time-resolved laser spectroscopic techniques due to the large penetration depth of the pump and probe pulses. Here, we use four-dimensional scanning ultrafast electron microscopy (4D S-UEM) with unique surface sensitivity to directly visualize the charge carrier dynamics on Si (100) crystals before and after surface treatment (that removes the native 
oxide layer) in real space and time simultaneously. Our time-resolved snapshots of the top surface and Kelvin probe-force microscopy results demonstrate that the oxide layer can be formed within minutes after surface treatment, creating undesirable surface-trap states that destroy the population of photogenerated charge carriers on the surface and possibly at the device interface. This new surface observation provides critical photophysical insights into how a few atomic layers of oxide can dramatically influence charge carrier recombination dynamics in silicon solar cells.

\section{TOC Graphic}

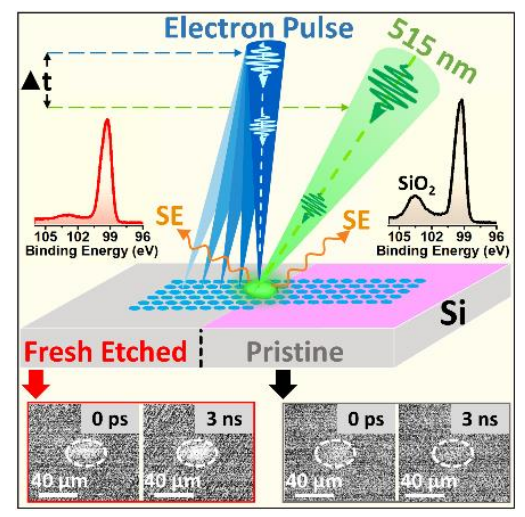


Crystalline silicon (c-Si) is the most dominant material in the semiconductor and photovoltaics (PV) industry. ${ }^{1}$ Si holds a major market share of more than $90 \%$ thanks to its stability, abundance, nontoxicity and high photo conversion efficiency. ${ }^{2-6}$ To date, the record Si single-junction cell has reached an efficiency record of $26.7 \%{ }^{7,8}$ to be compared to its theoretical limit of $\sim 29.4 \%{ }^{9}$ It is also a promising candidate for solar fuel generation, ${ }^{10-13}$ providing benchmarked efficiencies higher than $10 \%{ }^{14,15}$ Interfacial engineering is one of the few remaining frontiers for pushing the efficiency of Si solar cells closer to their theoretical efficiencies; as such it is essential to improve further our understanding of the fundamental photo physical processes at the nanoscale-surface level, which includes effects of light absorption, electron-hole separation and transport, and recombination through defects. ${ }^{3,16}$

Probing the lifetime of photo generated carriers is one of the most sensitive methods to assess material quality and holds direct relevance to device efficiency. ${ }^{16,17}$ For Si, due to its covalent nature, the surface often is the most important source of carrier recombination. Therefore, surface treatment and passivation have been essential preparation steps in the fabrication of all Si-based devices. ${ }^{18}$ The removal and protection of the surfaces from oxides, organic contamination and metallic impurities terminating the dangling bonds are crucial for achieving stability, reliability and high performance. ${ }^{18,19}$ The effective carrier lifetime $\left(\tau_{\text {eff }}\right)$ depends on both the bulk lifetime ( $\left.\tau_{\text {bulk }}\right)$ and surface lifetime $\left(\tau_{\text {surface }}\right)$. Generally, the time scale of $\tau_{\text {bulk }}$ mainly depends on the concentrations of carriers and doped atoms. ${ }^{20}$ Although $\tau_{\text {bulk }}$ can be calculated by theoretical models ${ }^{20}$, the results are usually higher than the $\tau_{\text {eff }}$ measured by relatively slow methods such as photoconductance techniques ${ }^{21}$ due to surface and interface carrier recombination. ${ }^{22}$ The differentiation between $\tau_{\text {bulk }}$ and $\tau_{\text {surface }}$ and how to directly measure them experimentally are among the largest challenges being faced by the materials science and physical chemistry 
communities. ${ }^{23-25}$ There have been several reports describing methods to determine $\tau_{\text {bulk }}$ and $\tau_{\text {surface }}$ in Si materials..$^{22,23,25,27-31}$ However, these approaches depend on mathematical models or only measuring $\tau_{\text {bulk }}$ by passivating the surface to eliminate surface carrier recombination and then calculate $\tau_{\text {surface }}$ with prior assumptions for the surface properties. In addition, the surface of silicon wafers controls the interface and contacting properties which often consist of stacks of different layers, often only a few nanometer thin, across which the transport of carriers occurs. $23,24,26,32,33$ Therefore, advanced techniques and strategies are urgently required to map the trap-densities, carrier lifetime and diffusion at the uppermost surfaces $\sim 1-5 \mathrm{~nm}$, especially in applications where the size of devices is scaling down to thin films and nanostructures.

A native oxide layer is grown on $\mathrm{Si}$ surfaces at room temperature whenever they are exposed to an environment of oxygen and moisture, even for a very short period. ${ }^{19}$ The thickness of this native oxide on the Si surface has been determined to be 1-2 $\mathrm{nm}$ or even in the angstrom range (few monolayers). ${ }^{35-40}$ Despite its thin thickness, this native oxide layer, like other surface contaminants, affects the band structure and the electronic properties of the host materials as mentioned in several reports. ${ }^{26,27,35,41}$ Therefore, much attention has been given to characterizing and controlling this native oxide including the effects of different etchants. ${ }^{35-42}$ Hydrofluoric acid (HF)-based solutions are the etchants most widely used for cleaning Si prior to any processing step. ${ }^{43-46}$ The HF solution removes Si oxides from the surface and terminates dangling bonds with hydrogen atoms to act as a passivation layer. ${ }^{35,36,43,44}$ Despite the usefulness of the HF-based cleaning, several angstroms of native oxide regrow on the etched clean surface within minutes. ${ }^{35-37,40}$ This reoxidation process becomes slower with time until reaching a saturation thickness. ${ }^{35,36}$ The atmosphere of the environment, especially the degree of moisture, affects the regrowth rate of this native oxide layer on the etched clean surfaces. ${ }^{36}$ Few reports have studied the surface recombination in HF-etched 
Si wafers using the optical pump-probe technique ${ }^{41}$ and lock-in carrierography. ${ }^{47}$ Sun et al. showed that the surface recombination rate varies with the amount of dissolved oxygen in the etchant solution and it increases as time passes after the etching. ${ }^{47}$ However, the large penetration depth of light, especially in $\mathrm{Si}(\sim 1330 \mathrm{~nm}$ at a light wavelength of $515 \mathrm{~nm})$, hinders the selectivity of discerning the native oxide layer that has at most a 2-nm thickness without the interference of the bulk properties when using a photon pump in the photon probe configuration. Four-dimensional scanning ultrafast electron microscopy (4D S-UEM) is the sole technique capable of the surfaceselective visualization of the light-triggered carrier dynamics at the angstrom-nanometer scale. ${ }^{48-}$ ${ }^{55} 4 \mathrm{D}$ S-UEM uses an electron probing system to scan the top surface of the photoactive materials after being pumped with a photon pulse. As a result, secondary electrons are ejected from the top few nanometers $(<5 \mathrm{~nm})$ and can be detected in real space and time. The carrier dynamics on $\mathrm{Si}$ surfaces have already been studied by $4 \mathrm{D}$ electron microscopy. ${ }^{56,57}$ However, no attention has been given to the effect of the native oxide layer on the surface charge carrier recombination.

In this work, 4D S-UEM is used to selectively study the Si surface, showing the effect of the native oxide layer (1-2 nm) in forming surface trap states that significantly further reduce the surface carrier lifetime. The uppermost surface dynamics are visualized before and after the HF-etching. In addition, the regrowth of the native oxide on HF-cleaned surface is observed by 4D S-UEM and Kelvin probe-force microscopy (KPFM). The results are of significance to understand the role of the native oxide in surface charge carrier recombination, together with the effect of etching, and represent a suitable case of study for 4D S-UEM.

A schematic representation of the principle of 4D S-UEM is given in Figure 1a. A pulsed primary electron beam is generated by a delayed UV excitation pulse $(343 \mathrm{~nm})$ from a cooled Schottky field-emitter tip, which generates secondary electrons (SEs) from the surface of the specimen. An 
optical laser pulse $(515 \mathrm{~nm})$ is used to excite the surface of the material for the carrier dynamics initiation. The SEs emitted from the material surface are analyzed pixel by pixel to construct timeresolved images at various well-defined time delays between the optical pulse and the electron pulse. SE difference images are formed by referencing the frames taken at different time delays to the one taken at a very far negative time when the electron pulse arrives prior to the photon pulse. The change in the SE intensity (i.e., image contrast) in the laser-irradiated region in contrast to the non-irradiated area reveals the surface carrier dynamics together with any surface defects. Figure $1 \mathrm{~b}$ shows time-resolved SE difference images of the Si surface with the native oxide layer (top) and the freshly stripped $\mathrm{Si}$ (bottom). At the far negative delays (e.g., -630 ps), no change in the

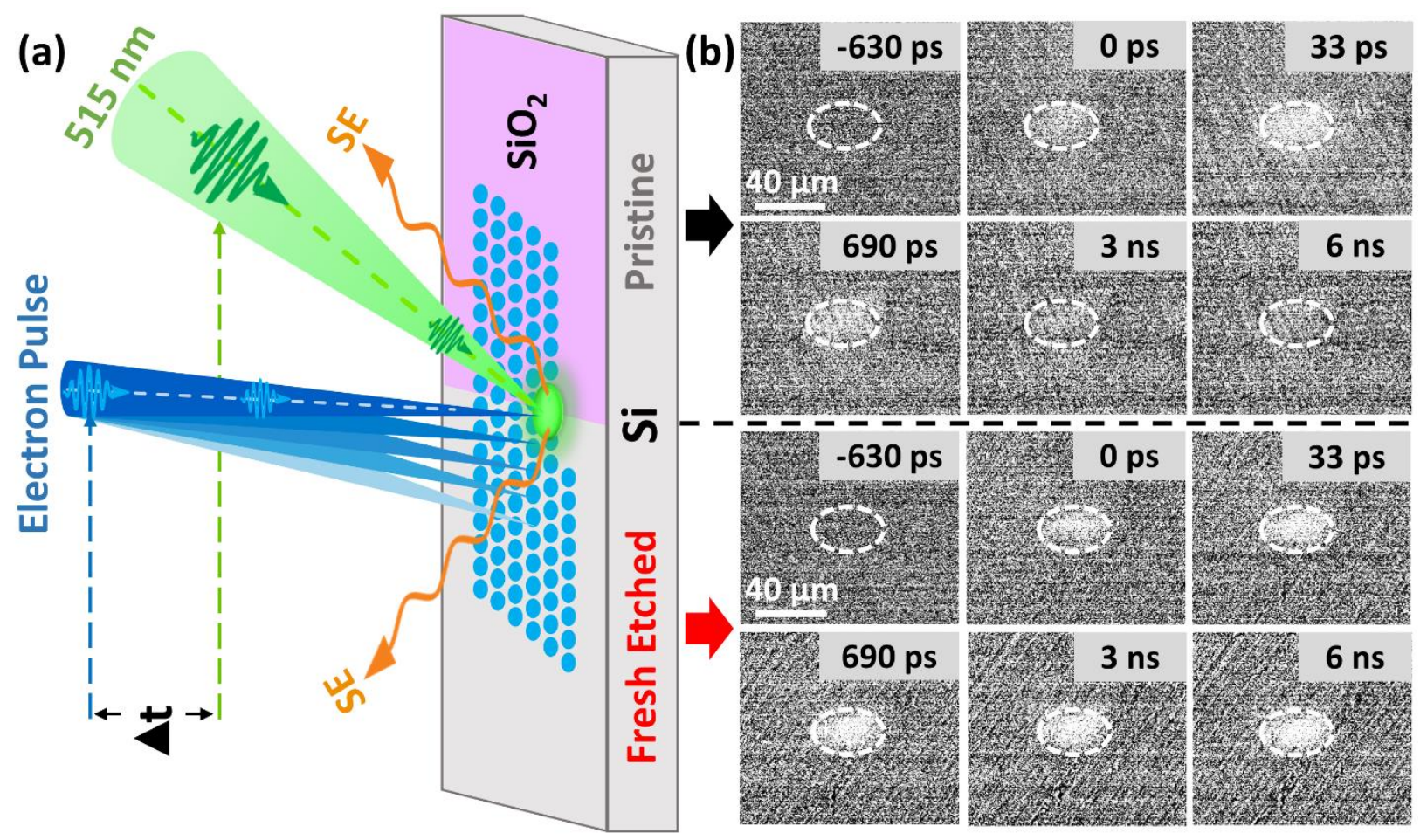

Figure 1. (a) Schematic of the 4D S-UEM principle. (b) Time-resolved SE difference images at indicated time delays for (top) pristine and (bottom) fresh HF-etched Si (100) in response to 515$\mathrm{nm}$ excitation. The dashed ellipse indicates the laser footprint on the sample. 
image contrast is observed, indicating the recovery of the system to the initial state after each pump-probe event. Starting from time zero (i.e., when the photon and electron pulses arrive at the same time) and at positive times (i.e., when the photon pulse arrives prior to the electron pulse), the SE intensity increases in the laser-irradiated region (dashed ellipse), resulting in a bright contrast in both samples. However, it is clear that this bright contrast fades very quickly in the pristine, as-cut $\mathrm{Si}$, while it remains until the end of our time delay window (i.e., $6 \mathrm{~ns}$ ) in the freshly etched sample.

The change in the SE intensity in the laser-irradiated region as a function of the time delay is plotted, as shown in Figure 2a. As observed in the SE difference images, the bright signal in the pristine sample rapidly disappears, indicating surface carrier trapping/recombination. The multilifetime components $(90 \pm 28 \mathrm{ps}, 5.5 \pm 1.1 \mathrm{~ns}$ in pristine $\mathrm{Si})$ could be attributed to the oxygen defect states and surface roughness as well as the inhomogeneity present on surfaces as shown in Figure S1. In contrast, the bright contrast in the HF-etched Si shows only a rise and then a longlived signal, indicating the absence of trapping centers in the bandgap of the Si (see Figure 1b, Figure $2 \mathrm{a})$. The slow rise $(180 \pm 52 \mathrm{ps})$ observed in the freshly etched sample could be attributed to the diffusion of photogenerated charges to the top surface. Experiments with detailed explanation for the origin of rise components observed in different materials at different applied voltages are beyond the scope of this work and will be published elsewhere.

This difference in the charge carrier recombination can be understood in terms of the electronic structure, as shown in the schematic of Figure $2 \mathrm{~b}$. Typically, when the Si is excited by a green photon pulse $(515 \mathrm{~nm}=2.4 \mathrm{eV})$, an interband carrier transition takes place, and electrons are promoted from the valence band to the conduction band with a higher probability of SE emission. 

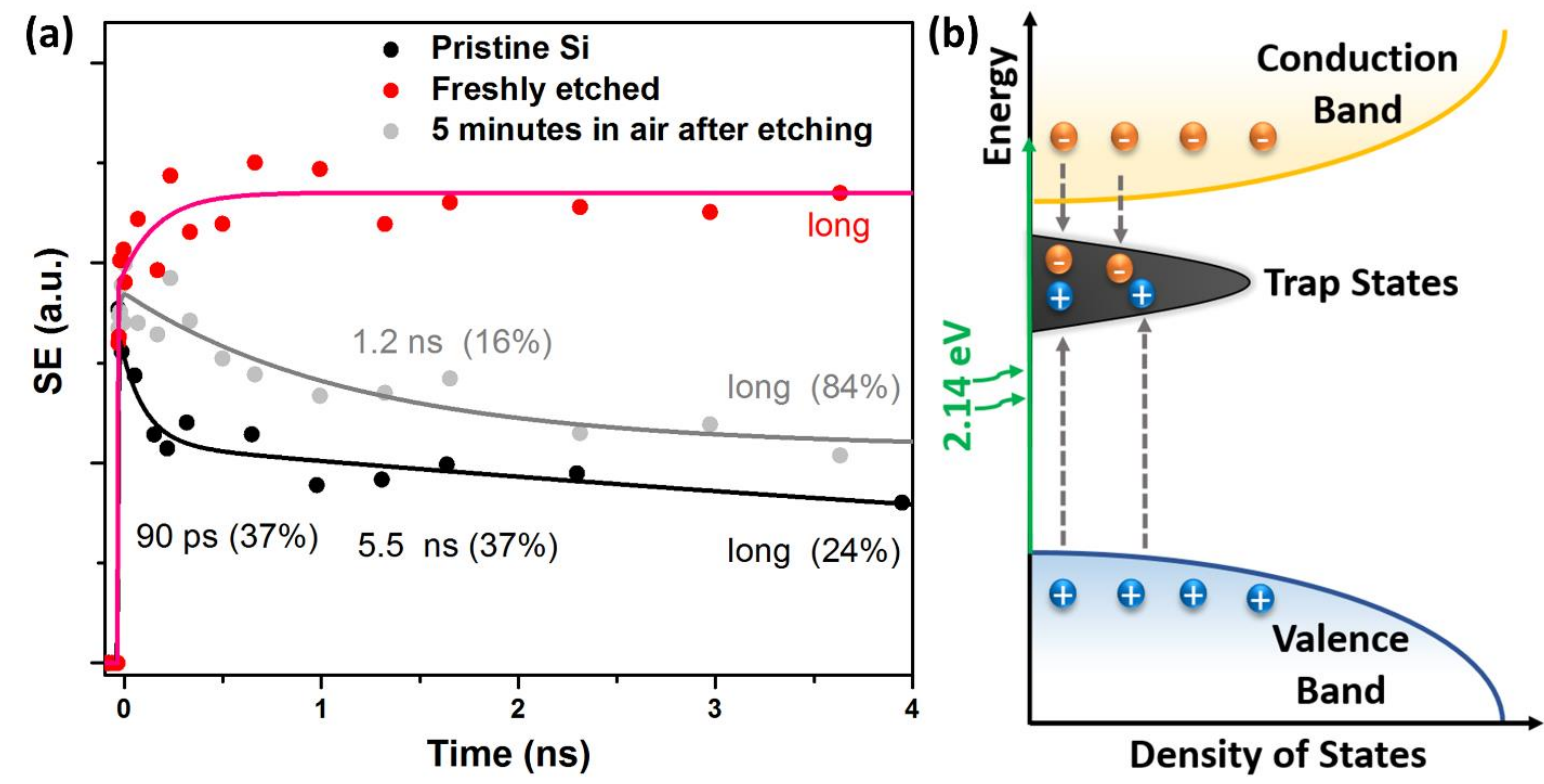

Figure 2. (a) Extracted kinetics of the time-resolved secondary electron, SE, intensities of the laser footprint region in the pristine $\mathrm{Si}$, freshly HF-etched $\mathrm{Si}$, and $\mathrm{Si}$ sample one day after HF-etching and exposure to air for 5 minutes. (b) Schematic of the density of states and the excitation and trapping processes.

Therefore, the increased SE in the irradiated region results in a bright-contrast formation. ${ }^{58,59}$ However, the presence of surface defects can act as trap states for the excited electrons, leading to faster recombination. ${ }^{55}$ This appears as a fast fading away of the bright signal. Since the only difference between the pristine and the freshly HF-etched Si is the thickness of the native oxide layer, we concluded that the trap states and the ultrafast carrier recombination here are due to the silicon-oxygen bonds (thin oxide layer). The same freshly HF-etched sample was kept in the SEM vacuum chamber and then exposed to air for a few minutes, and a re-measurement of this sample confirms the effect of the native oxide layer on the surface dynamics. As shown in Figure 2a, the dynamics in the etched sample left in air for 5 minutes are in between those of the pristine and freshly etched Si. The bright signal also fades away as in the pristine sample but the dynamics are much slower $(1.2 \pm 0.5 \mathrm{~ns})$. This means that trap states are quickly formed, although they are fewer 
than in the pristine sample. This clarifies the role of the native oxide layer in the charge carrier recombination of the Si surface.

X-ray photoelectron spectroscopy (XPS) has been used extensively to study the effect of HFetching on Si surfaces. ${ }^{37,41,44,60-62}$ However, here we investigated the surfaces of the pristine and freshly HF-etched Si by XPS to connect the information of the surface chemistry and the results obtained from the 4D S-UEM. The Si 2p, C 1s and O 1s XPS spectra of the pristine and freshly HF-etched Si are shown in Figure 3a on the same intensity axis. The Si $2 p$ spectrum of the pristine Si shows two peaks with binding energies of $99.3 \mathrm{eV}$ and $103.5 \mathrm{eV}$, which correspond to bulk Si and the surface oxide $\mathrm{SiO}_{2}$, respectively. ${ }^{37,40,60,61}$

(a)

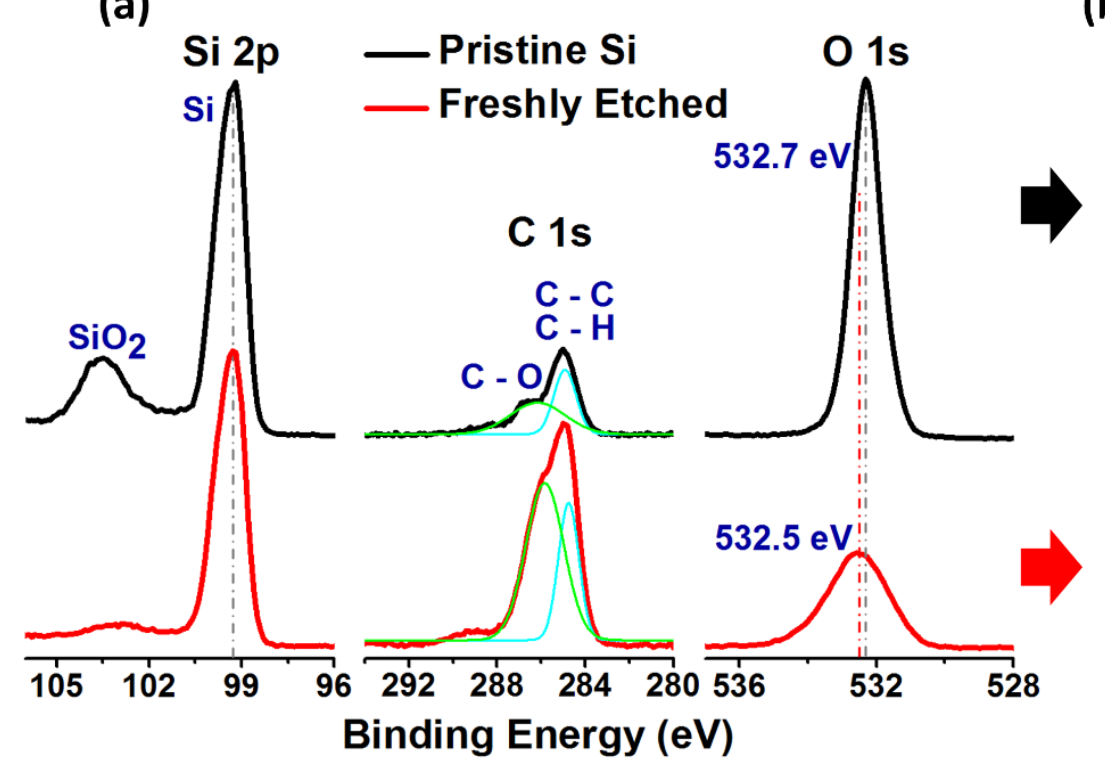

(b)
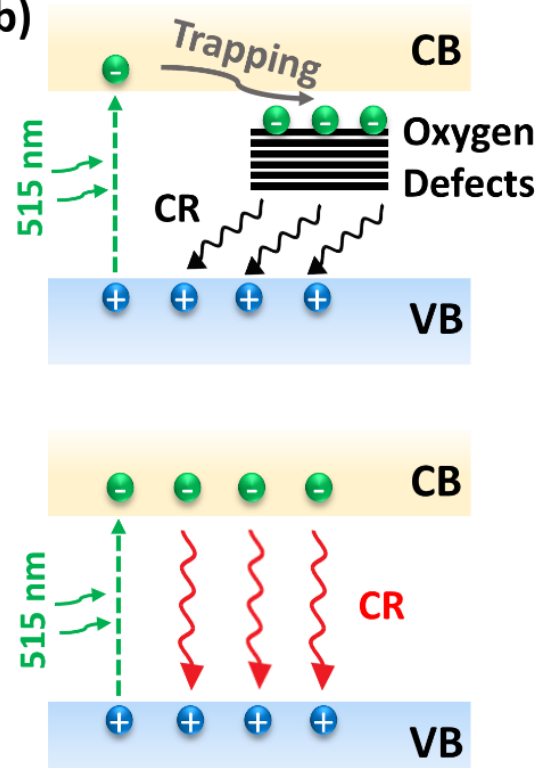

Figure 3. (a) XPS spectra of the pristine and freshly HF-etched $\mathrm{Si}$ with the same intensities in $\mathrm{Si}$ 2p, C 1s, and O 1s. (b) Illustration of the electronic structure before and directly after HF etching, showing the trapping caused by the oxygen defects.

In the freshly $\mathrm{HF}$-etched $\mathrm{Si}$, the $\mathrm{SiO}_{2}$ peak has almost disappeared. It is worth noting that the etching process by HF, and the XPS instrument are not located at the same site, so the XPS spectra were not obtained immediately after the etching but only after a transfer time, which may result in 
a slight increment in the oxidation level. The $\mathrm{C} 1 \mathrm{~s}$ spectrum can be deconvoluted into two peaks. ${ }^{63}$ In the pristine $\mathrm{Si}$, the $\mathrm{C}-\mathrm{C}$ and $\mathrm{C}-\mathrm{H}$ contamination peak is at $284.9 \pm 0.01 \mathrm{eV}$ and the $\mathrm{C}-\mathrm{O}$ peak is at $286.1 \pm 0.06 \mathrm{eV}$. In the freshly HF-etched $\mathrm{Si}$, the $\mathrm{C}-\mathrm{C}$ and $\mathrm{C}-\mathrm{H}$ peak is at $284.7 \pm 0.01 \mathrm{eV}$ and the $\mathrm{C}-\mathrm{O}$ peak is at $285.9 \pm 0.07 \mathrm{eV} \cdot{ }^{61}$ It is noted that the intensity of the $\mathrm{C} 1 \mathrm{~s}$ spectrum increases after etching, which is consistent with the literature. ${ }^{37}$ One possible explanation for this is that organic impurities are adsorbed on the Si surface during the deionized water rinse after etching. ${ }^{37}$ The $\mathrm{O} 1 \mathrm{~s}$ spectrum is composed of one peak at $532.7 \mathrm{eV}$ and $532.5 \mathrm{eV}$ for the pristine and freshly HF-etched $\mathrm{Si}$, respectively. The oxygen peak can be due to oxygen in contamination adsorbed on the surface and/or bound to the $\mathrm{Si}$ as $\mathrm{SiO}_{2} \cdot{ }^{37,64}$ After etching, the area under the oxygen peak is enormously reduced by $71 \%$. Besides, the $\mathrm{C}-\mathrm{O}$ peak is increased concluding that most of the left $\mathrm{O}$ after etching is bonded to $\mathrm{C}$ not Si. The XPS results together with the 4D S-UEM reveal the role of the $\mathrm{SiO}_{2}$ in forming surface trap states that in turn causes faster charge carrier recombination, as illustrated in Figure 3b.

Additionally, the surface potential in the air is measured by $\mathrm{KPFM}^{65}$ to highlight the regrowth of the native oxide as the air exposure time increases, as shown in Figure 4. A green laser (523 nm) is used to study the effect of illumination on the surface potential. The freshly HF-etched sample showed an increase in the surface potential upon illumination, indicating the formation of a high concentration of photo generated carriers on the surface, as shown in Figure 4a. However, the difference in the surface potential between the dark and illuminated states decreases as the measurement is repeated on the same sample. Until approximately 40 minutes, there is no difference between the surface potential measured at laser off and on, as shown in Figure $4 \mathrm{~b}$, indicating the regrowth of a layer of native oxide that again changes the surface properties. 


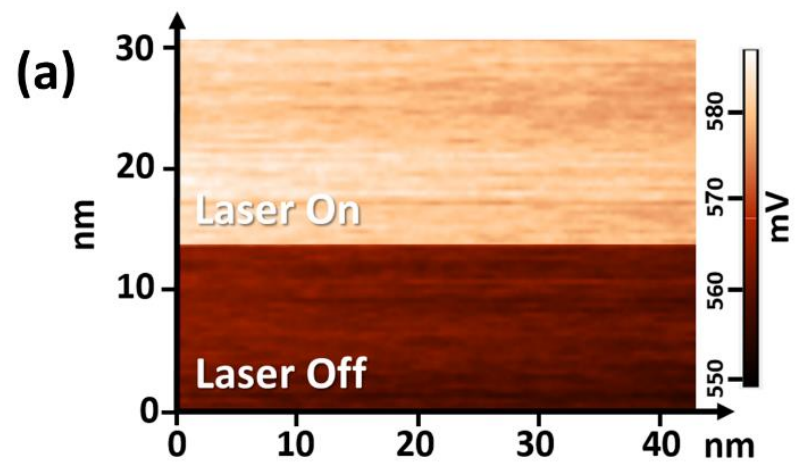

(d)
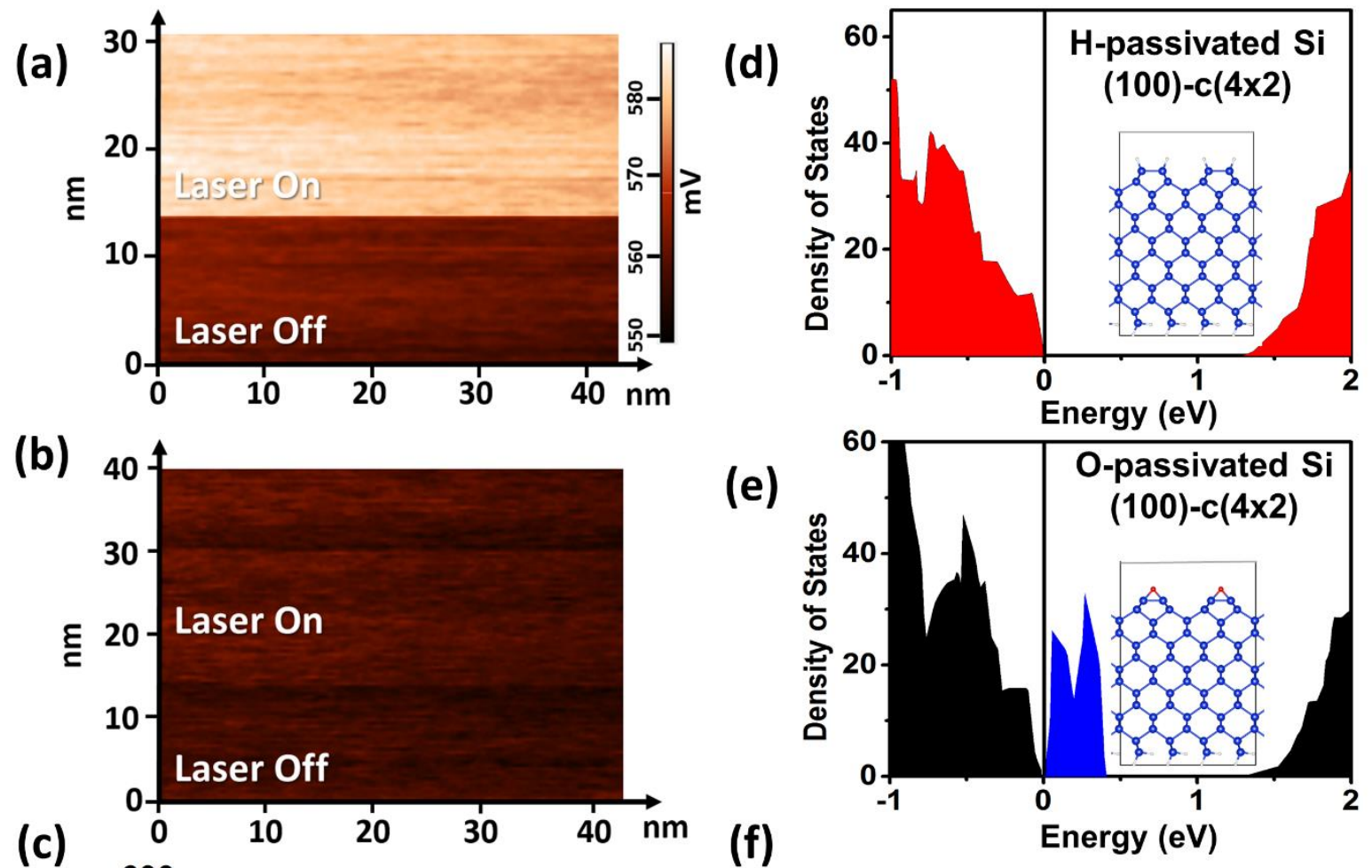

(e)
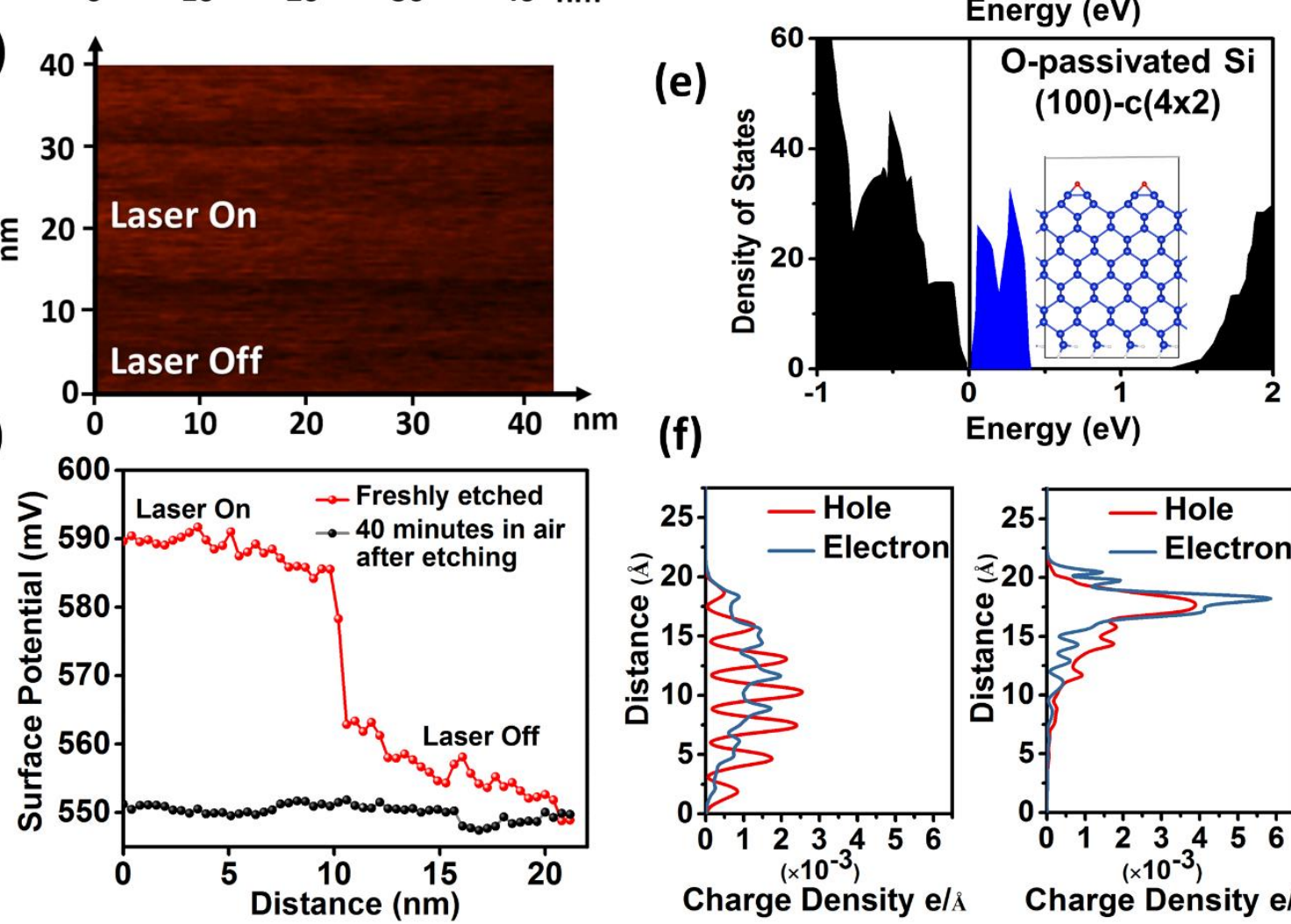

(f)
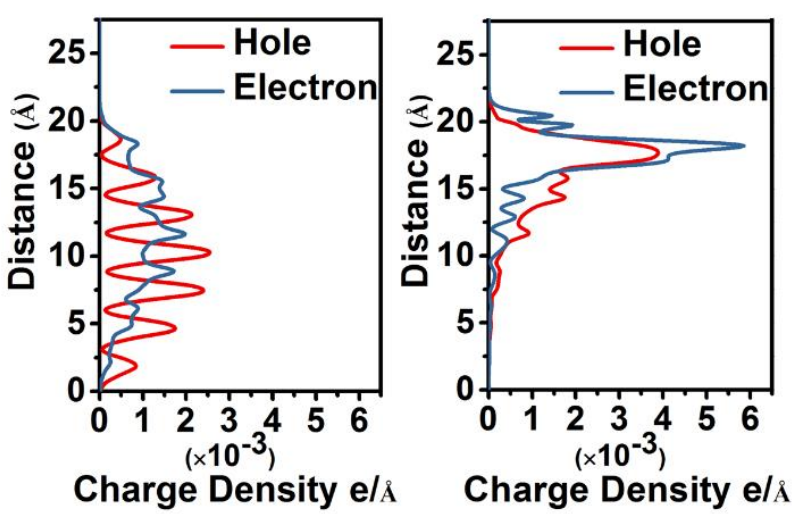

Figure 4. (a) KPFM image for freshly etched $\mathrm{Si}$ in the dark (bottom portion) and under the illumination of a 523-nm laser (top portion). (b) KPFM image for freshly HF-etched Si after 40 minutes from measurement in air showing no difference in the SP in the dark (bottom) and under 523-nm illumination (top). (c) Corresponding SP profiles of images (a) and (b). Optimized crystal structures and density of states (d) H-passivated Si (100)-c(4×2) and (e) O-passivated Si (100)$c(4 \times 2)$ slabs calculated at HSE level of theory (the blue area indicates the surface state). (f) Onedimensional plane-average electronic charge density for holes and electrons of $\mathrm{H}$-passivated $\mathrm{Si}$ $(100)$-c $(4 \times 2)$ (left) and O-passivated $\mathrm{Si}(100)-\mathrm{c}(4 \times 2)$ slabs (right). 
This result is in good agreement with the surface dynamics in Figure 2a. Figure 4c shows the corresponding surface potentials for the KPFM images. Density functional theory (DFT) calculations are performed to study the effects of $\mathrm{Si}(100)$ surface terminations on the charge density properties. The hybrid functional HSE is used in our study to correct the underestimated band gaps calculated at the GGA/PBE level of theory (see more computational details in the Experimental Section). Here, we consider four $\mathrm{Si}(100)-\mathrm{c}(4 \times 2)$ slab models $i)$ without passivation, ii) with full $\mathrm{H}$-passivation, iii) with full HO-passivation, and iv) with partial O-passivation, as shown in Figure 4d-f and Figure S2 in the Supporting Information. For the clean Si (100)-c(4×2) surface without passivation, the strong surface reconstruction (i.e., Si atom inward relaxation) leads to a significant surface state with holes and electrons accumulated on the top surface layers. Similarly, when the $\mathrm{Si}(100)-\mathrm{c}(4 \times 2)$ surface is partially passivated by $\mathrm{O}$ atoms, it retains high localized surface charges but increased charge densities, which results in charge carrier trapping on the Si (100) surface that decreases the charge carrier lifetime. However, once the Si (100)$\mathrm{c}(4 \times 2)$ surface is fully passivated by $\mathrm{H}$ atoms or $\mathrm{OH}$ - groups, the resulting slabs show delocalized charge density features for both holes and electrons along the whole slab.

In summary, the significant impact of the ultrathin native oxide layer on the surface-carrier dynamics of $\mathrm{Si}(100)$ is visualized simultaneously in space and time using 4D S-UEM. Despite the ultrathin thickness $(1-2 \mathrm{~nm})$ of the oxide layer making it impractical to probe by time-resolved optical techniques - 4D S-UEM was able to reveal the role of the layer in forming trap states that act as quenching centers, causing ultrafast charge carrier recombination on the surface of the $\mathrm{Si}$. More specifically, time-resolved SE images clearly demonstrate that the atomic surface layers of oxide control the overall charge recombination not only at the surface but also possibly at the interface of the Si absorber layer in solar cell devices. These 4D S-UEM observations were 
corroborated by Kelvin probe-force microscopy, XPS, and DFT. The experimental approach described in this work presents a strategy to assess the profound influence of seemingly superficial atomic oxide layers on semiconductor materials.

\section{EXPERIMENTAL METHODS}

Materials. Silicon samples were obtained from 6 inch phosphorous-doped silicon wafers (NorSun, resistivity $1-5 \mathrm{Ohm} / \mathrm{cm}$ ). The silicon samples were precleaned for 10 minutes at $90{ }^{\circ} \mathrm{C}$ in a standard piranha solution (sulfuric acid (98\%) and hydrogen peroxide (30\%), 3:1 by volume). To remove the native silicon oxide layer, the samples were immersed in a 5\% hydrofluoric acid solution for 30 seconds and subsequently rinsed three times in deionized water. Finally, the hydrophobic surface was dried with nitrogen. The samples were then transferred directly to the S-UEM vacuum chamber for performing the 4D measurements.

4D-SUEM Experimental Setup. The 4D S-UEM setup consisted of a femtosecond laser (ClarkMXR) operating at a wavelength of $1030 \mathrm{~nm}$ and a pulse width of $270 \mathrm{fs}$ integrated with a modified SEM (FEI Quanta 650). Briefly, the fundamental laser output was divided and directed into two independent harmonic generators (HGs) by a beam splitter to produce the second and third harmonic pulses. The output beam of the third harmonic was tightly focused on a cooled Schottky field-emitter tip (tungsten coated with zirconium oxide) to generate pulsed electrons. The second harmonic was focused on the surface of the specimen to initiate, i.e., clock, the dynamics. A computer-controlled optical delay line was used to control the relative delay between the electron and photon pulses. Upon the specimen excitation, secondary electrons (SEs) were emitted from the top surface of the sample and subsequently detected by a positively biased Everhart-Thornley detector. All experiments were done at a laser frequency of $8 \mathrm{MHz}$ and accelerating voltage of 30 
$\mathrm{keV}$. SE images were acquired by integrating 64 frames, with a dwell time of $300 \mathrm{~ns}$ for each pixel. A detailed description of the $4 \mathrm{D}$ imaging setup is provided elsewhere. ${ }^{50,59}$

X-Ray Photoelectron Spectroscopy (XPS). XPS measurements were done using an Axis Supra DLD spectrometer (Kratos Analytical) equipped with a monochromatic Al Ka X-ray source (hv $=$ $1486.6 \mathrm{eV}$ ) operating at $150 \mathrm{~W}$, a multichannel plate, and a delay line detector under a vacuum of 10-9 mbar. The binding energies were referenced to the C 1s binding energy of the adventitious carbon contamination, which was taken to be $285 \mathrm{eV}$ in all samples. The XPS measurements were performed on pristine samples, on which oxidation was predominant, and after HF-cleaning.

Surface Potential Measurements. Surface potential measurements were performed using scanning Kelvin microscopy and spreading resistance imaging using DCP30-type cantilevers (NT-MDT). The DCP30 is a Si cantilever with a gold-coated reflection side and a highly doped diamond coating ( $\sim 100 \mathrm{~nm}$ thickness) on the tip side, with a resonant frequency of approximately $350 \mathrm{kHz}$ and a force constant of approximately $35 \mathrm{~N} / \mathrm{m}$. Pristine and freshly etched samples were measured at room temperature in air.

\section{Computational Methods}

Density Functional Theory Calculations. Density functional theory (DFT) calculations were performed with a generalized gradient approximation (GGA)/Perdew-Burke-Ernzerhof (PBE) level using the projector-augmented wave (PAW) method, as implemented in the VASP code. ${ }^{66,67}$ For the bulk Si, a k-point grid of $6 \times 6 \times 6$ over the Brillouin zone was used during the crystal structure optimization and electronic property calculations. The electronic plane-wave cutoff energy was set to $500 \mathrm{eV}$. The resulting lattice constant of Si was a $=\mathrm{b}=\mathrm{c}=5.468 \AA$. Clean, $\mathrm{H}-$ terminated, HO-terminated, and O-terminated $\mathrm{Si}(100)$-c( $4 \times 2)$ slabs were constructed to study the 
surface electronic properties. ${ }^{68}$ All the crystal geometries were optimized until all forces on all atoms were smaller than $0.01 \mathrm{eV} / \AA$. The hybrid Heyd-Scuseria-Ernzerhof (HSE) functional with an exact exchange fraction of 0.18 was used to obtain the projected density of states and charge densities for holes and electrons.

\section{ASSOCIATED CONTENT}

Supporting Information. The following files are available free of charge. Static SEM image for Si (100) as-cut wafer. Optimized crystal structures, density of states and onedimensional plane-average electronic charge density for holes and electrons of $\mathrm{Si}(100)-\mathrm{c}(4 \times 2)$ slab models without passivation and with full HO-passivation. (PDF)

\section{AUTHOR INFORMATION}

\section{Corresponding Author}

*E-mail: omar.abdelsaboor@kaust.edu.sa.

\section{Notes}

The authors declare no competing financial interests.

\section{ACKNOWLEDGMENT}

The research reported in this publication was supported by funding from King Abdullah University of Science and Technology (KAUST).

\section{REFERENCES}

(1) Siffert, P.; Krimmel, E. F. Silicon Evolution and Future of a Technology; Springer: Berlin, Heidelberg, 2004.

(2) Andreani, L. C.; Bozzola, A.; Kowalczewski, P.; Liscidini, M.; Redorici, L., Silicon Solar Cells: Toward the Efficiency Limits. Adv. Phys:X. 2018, 4, 1-24.

(3) Liu, J.; Yao, Y.; Xiao, S.; Gu, X., Review of Status Developments of High-efficiency Crystalline Silicon Solar Cells. J. Phys. D: Appl. Phys. 2018, 51, 1-12. 
(4) Reese, M. O.; Glynn, S.; Kempe, M. D.; McGott, D. L.; Dabney, M. S.; Barnes, T. M.; Booth, S.; Feldman, D.; Haegel, N. M., Increasing Markets and Decreasing Package Weight for High-Specific-Power Photovoltaics. Nat. Energy 2018, 3, 1002-1012.

(5) Ribeyron, P.-J., Crystalline Silicon Solar Cells: Better than ever. Nat. Energy 2017, 2, 1-2.

(6) Battaglia, C.; Cuevas, A.; De Wolf, S., High-efficiency Crystalline Silicon Solar Cells: Status and Perspectives. Energy Environ. Sci. 2016, 9, 1552-1576.

(7) Yoshikawa, K.; Kawasaki, H.; Yoshida, W.; Irie, T.; Konishi, K.; Nakano, K.; Uto, T.; Adachi, D.; Kanematsu, M.; Uzu, H.; Yamamoto, K., Silicon Heterojunction Solar Cell with Interdigitated Back Contacts for a Photoconversion Efficiency Over 26\%. Nat. Energy 2017, 2, 1-8.

(8) Green, M. A.; Hishikawa, Y.; Dunlop, E. D.; Levi, D. H.; Hohl-Ebinger, J.; Yoshita, M.; Ho-Baillie, A. W. Y., Solar Cell Efficiency Tables (Version 53). Prog. Photovoltaics 2019, 27, 3-12.

(9) Richter, A.; Hermle, M.; Glunz, S. W., Reassessment of the Limiting Efficiency for Crystalline Silicon Solar Cells. IEEE J. Photovolt. 2013, 3, 1184-1191.

(10) Hou, F.; Yan, L.; Shi, B.; Chen, J.; Zhu, S.; Ren, Q.; An, S.; Zhou, Z.; Ren, H.; Wei, C.; Huang, Q.; Hou, G.; Chen, X.; Li, Y.; Ding, Y.; Wang, G.; Zhang, D.; Zhao, Y.; Zhang, X., Monolithic Perovskite/Silicon-Heterojunction Tandem Solar Cells with Open-Circuit Voltage of over 1.8 V. ACS Appl. Energy Mater. 2019, 2, 243-249.

(11) Luo, Z.; Wang, T.; Gong, J., Single-Crystal Silicon-based Electrodes for Unbiased Solar Water Splitting: Current Status and Prospects. Chem. Soc. Rev. 2019, DOI: $10.1039 / \mathrm{c} 8 \mathrm{cs} 00638 \mathrm{e}$.

(12) Mohamed, A. M.; Shaheen, B. S.; Mohammed, A. M.; Amer, A. W.; Allam, N. K. Recent Advances in the Use of Silicon-Based Photocathodes for Solar Fuel Production. In Advances in Silicon Solar Cells, Ikhmayies, S., Ed. Springer International Publishing: Cham, 2018; pp 229-267.

(13) Vijselaar, W.; Westerik, P.; Veerbeek, J.; Tiggelaar, R. M.; Berenschot, E.; Tas, N. R.; Gardeniers, H.; Huskens, J., Spatial Decoupling of Light Absorption and Catalytic activity of Ni-Mo-Loaded High-Aspect-Ratio Silicon Microwire Photocathodes. Nat. Energy 2018, 3, 185-192.

(14) Zheng, J.; Lyu, Y.; Wang, R.; Xie, C.; Zhou, H.; Jiang, S. P.; Wang, S., Crystalline TiO 2 Protective Layer with Graded Oxygen Defects for Efficient and Stable Silicon-based Photocathode. Nat. Commun. 2018, 9, 1-10.

(15) Schüttauf, J.-W.; Modestino, M. A.; Chinello, E.; Lambelet, D.; Delfino, A.; Dominé, D.; Faes, A.; Despeisse, M.; Bailat, J.; Psaltis, D.; Moser, C.; Ballif, C., Solar-to-Hydrogen Production at $14.2 \%$ Efficiency with Silicon Photovoltaics and Earth-Abundant Electrocatalysts. J. Electrochem. Soc. 2016, 163, F1177-F1181. 
(16) Wang, H.-P.; Sun, K.; Noh, S. Y.; Kargar, A.; Tsai, M.-L.; Huang, M.-Y.; Wang, D.; He, J.-H., High-Performance a-Si/c-Si Heterojunction Photoelectrodes for Photoelectrochemical Oxygen and Hydrogen Evolution. Nano Lett. 2015, 15, 2817-2824.

(17) Elani, U. A. I., The effective Carrier Lifetime Measurement in Silicon: The conductivity Modulation Method. J.K.S.U.S. 2010, 22, 9-13.

(18) Reinhardt, K.; Kern, W. Handbook of Silicon Wafer Cleaning Technology; William Andrew Applied Science Publishers, imprint of Elsevier: Kidlington, 2018.

(19) Morita, M.; Ohmi, T.; Hasegawa, E.; Kawakami, M.; Ohwada, M., Growth of Native Oxide on a Silicon Surface. J. Appl. Phys. 1990, 68, 1272-1281.

(20) Kerr, M. J.; Cuevas, A.; Sinton, R. A., Generalized Analysis of Quasi-Steady-State and Transient Decay Open Circuit Voltage Measurements. J. Appl. Phys. 2001, 91, 399-404.

(21) Sinton, R. A.; Cuevas, A., Contactless Determination of Current-Voltage Characteristics and Minority-Carrier Lifetimes in Semiconductors from Quasi-Steady-State Photoconductance Data. Appl. Phys. Lett. 1996, 69, 2510-2512.

(22) M'Saad, H.; Michel, J.; Lappe, J. J.; Kimerling, L. C., Electronic Passivation of Silicon Surfaces by Halogens. J. Electron. Mater. 1994, 23, 487-491.

(23) Giesecke, J. A.; Sinton, R. A.; Schubert, M. C.; Riepe, S.; Warta, W., Determination of Bulk Lifetime and Surface Recombination Velocity of Silicon Ingots From Dynamic Photoluminescence. IEEE J. Photovolt. 2013, 3, 1311-1318.

(24) Wu, B.; Nguyen, H. T.; Ku, Z.; Han, G.; Giovanni, D.; Mathews, N.; Fan, H. J.; Sum, T. C., Discerning the Surface and Bulk Recombination Kinetics of Organic-Inorganic Halide Perovskite Single Crystals. Adv. Energy Mater. 2016, 6, 1-9.

(25) Turek, M., Influence Of Bulk And Surface Properties On Measurable Steady-State Carrier-Lifetime. Energy Procedia 2012, 27, 33-37.

(26) Grant, N. E.; McIntosh, K. R.; Tan, J. T., Evaluation of the Bulk Lifetime of Silicon Wafers by Immersion in Hydrofluoric Acid and Illumination. ECS J. Solid State Sci. Technol. 2012, 1, 55-61.

(27) Ogita, Y. I., Bulk Lifetime and Surface Recombination Velocity Measurement Method in Semiconductor Wafers. J. Appl. Phys. 1996, 79, 6954-6960.

(28) Richter, A.; Glunz, S. W.; Werner, F.; Schmidt, J.; Cuevas, A., Improved Quantitative Description of Auger Recombination in Crystalline Silicon. Phys. Rev. B 2012, 86, 1-14.

(29) Ryu, K.; Choi, C.-J.; Rohatgi, A.; Ok, Y.-W., Study of Degradation in Bulk Lifetime of N-type Silicon Wafer due to Oxidation of Boron-rich Layer. Curr. Appl. Phys. 2016, 16, $497-500$. 
(30) Wang, K.; Kampwerth, H., A Method to Separate Bulk Lifetime and Surface Recombination Velocity of Silicon Bricks based on Transient Photoluminescence. Energy Procedia 2014, 55, 161-168.

(31) Wang, K.; Kampwerth, H., Separation Algorithm for Bulk Lifetime and Surface Recombination Velocity of Thick Silicon Wafers and Bricks via Time-Resolved Photoluminescence Decay. J. Appl. Phys. 2014, 115, 1-5.

(32) Schmidt, J.; Peibst, R.; Brendel, R., Surface Passivation of Crystalline Silicon Solar Cells: Present and Future. Sol. Energy Mat. Sol. C. 2018, 187, 39-54.

(33) Yao, B.; Zhang, J.; Fan, X.; He, J.; Li, Y., Surface Engineering of Nanomaterials for Photo-Electrochemical Water Splitting. Small 2019, 15, 1-20.

(34) Chen, L.; Xiao, C.; He, X.; Yu, B.; Kim, S. H.; Qian, L., Friction and Tribochemical Wear Behaviors of Native Oxide Layer on Silicon at Nanoscale. Tribol. Lett. 2017, 65, 1-8.

(35) Liu, W.; Meng, F.; Zhang, X.; Liu, Z., Evolution of a Native Oxide Layer at the a-Si:H/cSi Interface and Its Influence on a Silicon Heterojunction Solar Cell. ACS Appl. Mater. Interfaces 2015, 7, 26522-26529.

(36) Miura, T.-a.; Niwano, M.; Shoji, D.; Miyamoto, N., Initial Stages of Oxidation of Hydrogen-terminated Si Surface Stored in Air. Appl. Surf. Sci. 1996, 100-101, 454-459.

(37) Raider, S. I.; Flitsch, R.; Palmer, M. J., Oxide Growth on Etched Silicon in Air at Room Temperature. J. Electrochem. Soc. 1975, 122, 413-418.

(38) Shi, Y.; Han, T.; Gimbert-Suriñach, C.; Song, X.; Lanza, M.; Llobet, A., Substitution of Native Silicon Oxide by Titanium in Ni-coated Silicon Photoanodes for Water Splitting Solar Cells. J. Mater. Chem. A 2017, 5, 1996-2003.

(39) Tao, Y.; Hauert, R.; Degen, C. L., Exclusively Gas-Phase Passivation of Native OxideFree Silicon (100) and Silicon (111) Surfaces. ACS Appl. Mater. Interfaces 2016, 8, 1315713165.

(40) Zazzera, L. A.; Moulder, J. F., XPS and SIMS Study of Anhydrous HF and UV/OzoneModified Silicon (100) Surfaces. J. Electrochem. Soc. 1989, 136, 484-491.

(41) Sabbah, A. J.; Riffe, D. M., Measurement of Silicon Surface Recombination Velocity Using Ultrafast Pump-Probe Reflectivity in the Near Infrared. J. Appl. Phys. 2000, 88, 6954-6956.

(42) Neuwald, U.; Hessel, H. E.; Feltz, A.; Memmert, U.; Behm, R. J., Initial Stages of Native Oxide Growth on Hydrogen Passivated Si(111) Surfaces Studied by Scanning Tunneling Microscopy. Appl. Phys. Lett. 1992, 60, 1307-1309.

(43) Deegan, T.; Hughes, G., An X-ray Photoelectron Spectroscopy Study of the HF Etching of Native Oxides on Ge(111) and Ge(100) Surfaces. Appl. Surf. Sci. 1998, 123-124, 66-70. 
(44) Huang, L. J.; Lau, W. M., Effects of HF Cleaning and Subsequent Heating on the Electrical Properties of Silicon (100) Surfaces. Appl. Phys. Lett. 1992, 60, 1108-1110.

(45) Kang, J. K.; Musgrave, C. B., The mechanism of $\mathrm{HF} / \mathrm{H}_{2} \mathrm{O}$ Chemical Etching of $\mathrm{SiO}_{2}$. J. Chem. Phys. 2001, 116, 275-280.

(46) Shvartsev, B.; Gelman, D.; Komissarov, I.; Epshtein, A.; Starosvetsky, D.; Ein-Eli, Y., Influence of Solution Volume on the Dissolution Rate of Silicon Dioxide in Hydrofluoric Acid. ChemPhysChem 2015, 16, 370-376.

(47) Sun, Q. M.; Melnikov, A.; Mandelis, A.; Pagliaro, R., Surface Recombination Velocity Imaging of HF-Etched Si Wafers Using Dynamic Heterodyne Lock-In Carrierography. Solid State Phenom. 2018, 282, 13-18.

(48) Adhikari, A.; Eliason, J. K.; Sun, J.; Bose, R.; Flannigan, D. J.; Mohammed, O. F., FourDimensional Ultrafast Electron Microscopy: Insights into an Emerging Technique. ACS Appl. Mater. Interfaces 2017, 9, 3-16.

(49) Mohammed, O. F.; Yang, D.-S.; Pal, S. K.; Zewail, A. H., 4D Scanning Ultrafast Electron Microscopy: Visualization of Materials Surface Dynamics. J. Am. Chem. Soc. 2011, 133, 7708-7711.

(50) Sun, J.; Adhikari, A.; Shaheen, B. S.; Yang, H.; Mohammed, O. F., Mapping Carrier Dynamics on Material Surfaces in Space and Time using Scanning Ultrafast Electron Microscopy. J. Phys. Chem. Lett. 2016, 7, 985-994.

(51) Yang, D.-S.; Mohammed, O. F.; Zewail, A. H., Scanning Ultrafast Electron Microscopy. Proc. Natl. Acad. Sci. U.S.A. 2010, 107, 14993-14998.

(52) Bose, R.; Adhikari, A.; Burlakov, V. M.; Liu, G.; Haque, M. A.; Priante, D.; Hedhili, M. N.; Wehbe, N.; Zhao, C.; Yang, H.; Ng, T. K.; Goriely, A.; Bakr, O. M.; Wu, T.; Ooi, B. S.; Mohammed, O. F., Imaging Localized Energy States in Silicon-Doped InGaN Nanowires Using 4D Electron Microscopy. ACS Energy Lett. 2018, 3, 476-481.

(53) Bose, R.; Bera, A.; Parida, M. R.; Adhikari, A.; Shaheen, B. S.; Alarousu, E.; Sun, J.; Wu, T.; Bakr, O. M.; Mohammed, O. F., Real-Space Mapping of Surface Trap States in CIGSe Nanocrystals Using 4D Electron Microscopy. Nano Lett. 2016, 16, 4417-4423.

(54) Shaheen, B. S.; Sun, J.; Yang, D.-S.; Mohammed, O. F., Spatiotemporal Observation of Electron-Impact Dynamics in Photovoltaic Materials Using 4D Electron Microscopy. $J$. Phys. Chem. Lett. 2017, 8, 2455-2462.

(55) El-Zohry, A. M.; Shaheen, B. S.; Burlakov, V. M.; Yin, J.; Hedhili, M. N.; Shikin, S.; Ooi, B.; Bakr, O. M.; Mohammed, O. F., Extraordinary Carrier Diffusion on CdTe Surfaces Uncovered by 4D Electron Microscopy. Chem 2019, 5, 706-718.

(56) Liao, B.; Najafi, E.; Li, H.; Minnich, A. J.; Zewail, A. H., Photo-Excited Hot Carrier Dynamics in Hydrogenated Amorphous Silicon Imaged by 4D Electron Microscopy. Nat. Nanotechnol. 2017, 12, 871-877. 
(57) Najafi, E.; Ivanov, V.; Zewail, A.; Bernardi, M., Super-Diffusion of Excited Carriers in Semiconductors. Nat. Commun. 2017, 8, 1-7.

(58) Cho, J.; Hwang, T. Y.; Zewail, A. H., Visualization of Carrier Dynamics in p(n)-type GaAs by Scanning Ultrafast Electron Microscopy. Proc. Natl. Acad. Sci. U.S.A. 2014, 111, 2094-2099.

(59) Sun, J.; Melnikov, V. A.; Khan, J. I.; Mohammed, O. F., Real-Space Imaging of Carrier Dynamics of Materials Surfaces by Second-Generation Four-Dimensional Scanning Ultrafast Electron Microscopy. J. Phys. Chem. Lett. 2015, 6, 3884-3890.

(60) Morita, M.; Ohmi, T., Characterization and Control of Native Oxide on Silicon. Jpn. J. Appl. Phys. 1994, 33, 370-374.

(61) Thøgersen, A.; Selj, J. H.; Marstein, E. S., Oxidation Effects on Graded Porous Silicon Anti-Reflection Coatings. J. Electrochem. Soc. 2012, 159, D276-D281.

(62) Weinberger, B. R.; Peterson, G. G.; Eschrich, T. C.; Krasinski, H. A., Surface Chemistry of HF Passivated Silicon: X-ray Photoelectron and Ion Scattering Spectroscopy Results. J. Appl. Phys. 1986, 60, 3232-3234.

(63) Bashouti, M.; Ristein, J.; Haick, H.; Christiansen, S., A Non-Oxidative Approach Towards Hybrid Silicon Nanowire-Based Solar Cell Heterojunctions. HYMA. 2013, 1, 214.

(64) Radvanyi, E.; De Vito, E.; Porcher, W.; Jouanneau Si Larbi, S., An XPS/AES Comparative Study of the Surface Behaviour of Nano-Silicon Anodes for Li-Ion Batteries. J. Anal. Atom. Spectrom. 2014, 29, 1120-1131.

(65) Yan, F.; Chen, G.; Lu, L.; Spanier, J. E., Dynamics of Photogenerated Surface Charge on $\mathrm{BiFeO}_{3}$ Films. ACS Nano 2012, 6, 2353-2360.

(66) Kresse, G.; Furthmüller, J., Efficiency of ab-initio Total Energy Calculations for Metals and Semiconductors Using a Plane-Wave Basis Set. Comp. Mater. Sci. 1996, 6, 15-50.

(67) Kresse, G.; Joubert, D., From Ultrasoft Pseudopotentials to the Projector AugmentedWave Method. Phys. Rev. B 1999, 59, 1758-1775.

(68) Dürr, M.; Höfer, U., Dissociative Adsorption of Molecular Hydrogen on Silicon Surfaces. Surf. Sci. Rep. 2006, 61, 465-526. 